\title{
Raymond Torres*
}

\section{POLÍTICA FISCAL EUROPEA: SITUACIÓN Y PERSPECTIVAS DE REFORMA}

\section{Los países que pertenecen a la eurozona se enfrentan a serios desafios en su política}

fiscal. En el actual sistema europeo de coordinación de políticas nacionales, las políticas fiscales tienden a ser procíclicas, lo que agudiza los problemas coyunturales, afecta el potencial de crecimiento y dificulta el desendeudamiento. Por otra parte, Europa carece de instrumentos capaces de reaccionar a choques asimétricos y corregir divergencias persistentes de productividad como las que se están produciendo. Existen opciones de reforma y de potenciación de una política fiscal europea, que suponen un abandono de soberanía nacional a cambio de una mayor eficacia y más solidaridad.

Palabras clave: fiscalidad internacional, política monetaria, planificación y reforma.

Clasificación JEL: D31, F41, H31, H87.

\section{Introducción}

Desde la creación de la moneda única y el abandono de la soberanía monetaria nacional, los Gobiernos de la eurozona solo disponen del instrumento presupuestario para incidir sobre la coyuntura y hacer frente a eventuales choques. Así pues, la política fiscal se ha convertido en el principal pilar de gestión macroeconómica de esos países.

En teoría, la política fiscal puede apoyar la coyuntura en momentos de recesión y emprender el camino de la consolidación cuando la expansión se ha afianzado.

\footnotetext{
* Director de Coyuntura y Economía Internacional, FUNCAS.

El autor agradece la ayuda de Patricia Sánchez Juanino en la preparación de los gráficos.
}

Sin embargo, en la práctica, la política fiscal no ha jugado el papel contracíclico que cabía esperar'1.

Por una parte, desde el inicio de la crisis de la deuda soberana en 2010, la Unión Europea ha fortalecido los mecanismos de seguimiento y coordinación de la política fiscal de los países miembros, especialmente dentro de la eurozona. Este proceso se caracteriza por un esfuerzo de contención de los déficits, a partir de una vigilancia estrecha de las finanzas públicas de cada país. El endurecimiento del Pacto de Estabilidad y Crecimiento (particularmente con la adopción del «compacto fiscal») representa un importante pilar de esta política (Begg, 2018).

\footnotetext{
1 Véase el Informe anual del Consejo Fiscal Europeo (European Fiscal Board, 2017).
} 
Más recientemente, el debate contempla un giro en la política fiscal europea. Ahora se consideran acciones supranacionales como la creación de un instrumento europeo de gestión anticíclica, y un seguimiento de las políticas nacionales que tiene en cuenta la posición fiscal del conjunto de la UE. Pero todavía se trata de ideas y proyectos, más que de realizaciones concretas.

El objetivo de este artículo es analizar la situación y las perspectivas de la política europea en materia fiscal. En primer lugar, el artículo revisa los mecanismos existentes, tanto desde el punto de vista de la coordinación de las políticas de cada país como del lanzamiento de nuevos instrumentos a nivel europeo. En segundo lugar, el artículo examina los impactos, positivos y negativos, de los mecanismos existentes sobre los desequilibrios presupuestarios, el crecimiento y el empleo. El análisis es esencialmente cualitativo, aunque también se apoya en la observación de los principales indicadores así como en los resultados de varios estudios. Finalmente, el artículo presenta opciones de reforma. El debate que se ha abierto se centra ahora en dos frentes principales: por un lado, establecer mecanismos para reforzar la coordinación de las políticas fiscales nacionales, que incluye aspectos como la posibilidad de simplificar el pacto de estabilidad, armonizar bases impositivas, coordinación frente a la evasión fiscal; por otro lado, establecer mecanismos fiscales de ámbito europeo, como esquemas europeos de protección de inversiones, de seguro/reaseguro del desempleo, un fondo para contingencias extraordinarias (rainy day fund), o propuestas más ambiciosas que pasan por crear un Tesoro para la eurozona con capacidad de desarrollar una política fiscal anticíclica.

\section{Coordinación europea de las políticas fiscales nacionales}

El actual método de coordinación de política fiscal entre países europeos se basa en decisiones adoptadas a partir de 2011, en plena crisis de la deuda soberana. La crisis financiera que generó la caída de Lehman Brothers en 2008 afectó tanto a la economía europea como a la de la mayoría de países, tanto desarrollados como emergentes. Como respuesta a la crisis financiera, los países europeos se unieron al esfuerzo global para salir de la recesión mediante medidas de estímulo fiscal. Así pues, el G20 decidió un apoyo fiscal a la recuperación, por un monto de en torno al 2 por 100 del PIB mundial ${ }^{2}$. Como consecuencia de los estímulos, los déficits públicos se dispararon y con ellos la deuda pública. Esta última también se disparó por el apoyo al sector financiero.

Los primeros «brotes verdes» de la recuperación se manifestaron a partir de principios de 2010. Ese año, la economía volvió a crecer en todos los países desarrollados, salvo Grecia y Letonia. En España también avanzó el PIB (aunque apenas una centésima), pese al lastre que representaba el estallido de la burbuja inmobiliaria y después de la fuerte contracción de la actividad sufrida en 2009.

A partir de 2010, la Comisión Europea recomienda un giro en la política fiscal hacia una posición restrictiva. Su análisis, que minimiza los riesgos de fragmentación financiera inherentes a la eurozona, parte de la premisa de que una política macroeconómica restrictiva no afectará a la recuperación. El grueso del sistema actual de seguimiento y coordinación de políticas fiscales nacionales radica en ese período. En este apartado empezaremos por describir ese sistema, para después examinar sus principales impactos.

\section{El sistema actual contiene acciones preventivas y correctivas}

La coordinación europea de políticas fiscales nacionales consiste en un complejo entramado de normas, recomendaciones y códigos de conducta (Wieser, 2018).

En primer lugar, a partir de su reformulación de 2011 (Regulación 1173/2011), el Pacto de Estabilidad

\footnotetext{
2 Ver FMI (2009). Declaración del G20 de Londres.
} 
y Crecimiento prevé acciones preventivas para evitar déficits públicos excesivos.

Estas acciones se basan en el reconocimiento de la interconexión entre las economías que participan en la unión económica y monetaria, del que se deriva la necesidad de una vigilancia multilateral de las políticas macroeconómicas por parte de la Comisión Europea (artículo 121 del Tratado de Funcionamiento de la Unión Europea).

Esta vigilancia preventiva se organiza en torno a tres pilares. En primer lugar, el objetivo a medio plazo es de un equilibrio estructural de las finanzas públicas. Así pues, el objetivo de déficit, corregido de la evolución del ciclo, no debe superar el 1 por 100 del PIB. El valor específico del objetivo se define cada tres años (pero siempre con el límite del 1 por 100), o incluso con mayor periodicidad en función de las reformas estructurales adoptadas.

En segundo lugar, cada mes de abril los países de la eurozona se someten a un programa de estabilidad con los objetivos a medio plazo y las principales hipótesis. El Consejo examina el programa junto con una evaluación de la Comisión, que tiene en cuenta la senda prevista para el gasto público. Seguidamente, el Consejo emite una opinión con recomendaciones que forman parte del llamado Semestre Europeo. Así pues, el Consejo puede pedir ajustes con respecto al programa de estabilidad.

Finalmente, en caso de no cumplir con esos ajustes, el país se expone a sanciones de hasta el 0,2 por 100 del PIB.

Además de las medidas preventivas, el Pacto de Estabilidad y Crecimiento dispone que cuando un país se aleja del equilibrio, poniendo en peligro el buen funcionamiento del conjunto de la Unión, el Consejo puede examinar el caso y proponer medidas correctivas. En este caso, el Consejo está autorizado a tomar decisiones sin tener en cuenta el voto del país examinado. Así pues, el Pacto incluye un procedimiento por déficit excesivo (PDE), que es una medida de carácter correctivo ${ }^{3}$. EI PDE se activa cuando los desequilibrios de las cuentas de las Administraciones Públicas exceden ciertos criterios. Esos consisten en un límite de déficit del 3 por 100 del PIB y en una senda de desendeudamiento, cuando este supera el 60 por 100 del PIB.

La interpretación sobre el grado de cumplimiento de estos criterios está en manos del Consejo, en base a un informe de la Comisión. El Consejo puede pedir a un Estado la adopción de medidas correctivas en un plazo de menos de seis meses. En caso de no cumplimiento reiterado, el Consejo puede recurrir a sanciones, que pueden llegar hasta el 0,5 por 100 del PIB del país que contraviene los criterios. Existe la posibilidad de imponer sanciones adicionales en caso de obstáculos al mecanismo de seguimiento o de manipulación de las estadísticas.

Finalmente, el Pacto Fiscal (Fiscal Compact) que entró en vigor en 2013 establece una "regla de oro» para el déficit público, que no puede exceder el 0,5 por 100 el PIB a lo largo de un ciclo (1 por 100 para los países poco endeudados). La regla de oro es vinculante para países que han ratificado el Pacto (es decir, todos los miembros de la Unión Europea, salvo Croacia, Reino Unido y República Checa). Esto significa que el objetivo de déficit debe figurar en la ley o en la carta magna de cada país.

En el caso de España, el principio de equilibrio presupuestario está establecido en el artículo 135 de la Constitución. Asimismo, la ley de estabilidad presupuestaria establece una «regla de gasto», que limita el incremento de los empleos no financieros de las Administraciones Públicas al crecimiento del PIB nominal.

En principio, un Estado miembro puede recurrir ante el Tribunal de Justicia de la Unión Europea si estima que otro Estado miembro no respeta la regla de oro. Por otra parte, el apoyo financiero del Mecanismo Europeo

\footnotetext{
3 EI PDE también se fundamenta en el Pacto de Estabilidad y Crecimiento (art. 126 del Tratado de Funcionamiento de la Unión Europea), y por la norma adoptada en 2011 (Regulación 1173/2011)
} 


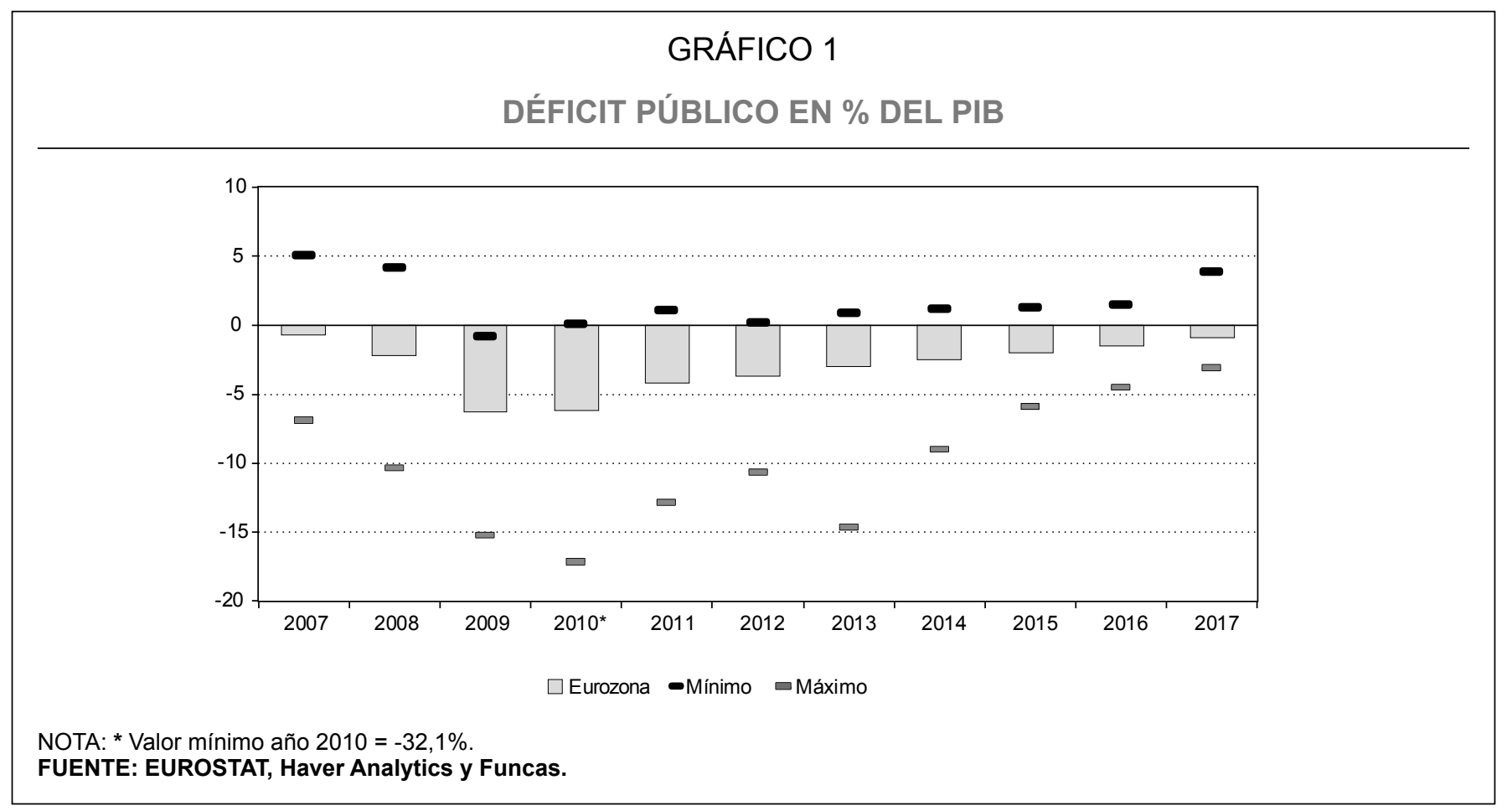

de Estabilidad (MEDE) está reservado a los países que han ratificado el pacto fiscal.

\section{La coordinación fiscal europea se enfrenta a importantes desafíos}

El endurecimiento de las normas europeas de política fiscal iniciado en plena crisis de la deuda, sobre todo a partir de las regulaciones de 2011 y del pacto fiscal de 2013, ha ido de la mano de una cierta reducción de los desequilibrios. Para el conjunto de los países europeos, el déficit público ha seguido una senda descendente, sobre todo a partir de 2013 (Gráfico 1). Sin duda, la reducción de tipos de interés, de la mano de la intervención del BCE ha contribuido a ese resultado. No obstante, el déficit primario (que descuenta el pago de intereses) también se ha reducido.

Igualmente, se ha instalado una cultura de seguimiento de las grandes magnitudes presupuestarias, con una cierta repercusión tanto en las decisiones de los parlamentos nacionales en materia presupuestaria como en el debate público. Por otra parte, los mecanismos europeos han abierto un espacio de diálogo entre Gobiernos, Comisión Europea y expertos académicos.

No obstante, el marco europeo de coordinación de políticas fiscales se enfrenta a importantes desafíos. El principal es el carácter procíclico de los ajustes que se derivan del mecanismo de seguimiento (Gráfico 2). Esto es importante porque una política fiscal procíclica -además de desaprovechar el único instrumento de gestión macroeconómica de que disponen los países de la eurozona - tiende a agravar las recesiones, a producir más paro y a reducir el crecimiento potencial. Así pues, algunos autores han detectado un impacto adverso de este tipo de políticas sobre la economía europea, tanto a corto plazo como en términos de crecimiento a largo plazo (Fatás y Summers, 2017).

Es un hecho que la política fiscal se ha endurecido durante la fase de debilitamiento provocada por la crisis de la deuda (Bénassy-Quéré et al., 2018). 


\section{GRÁFICO 2}

\section{LA POLÍTICA FISCAL EN LA EUROZONA HA SIDO PROCÍCLICA}

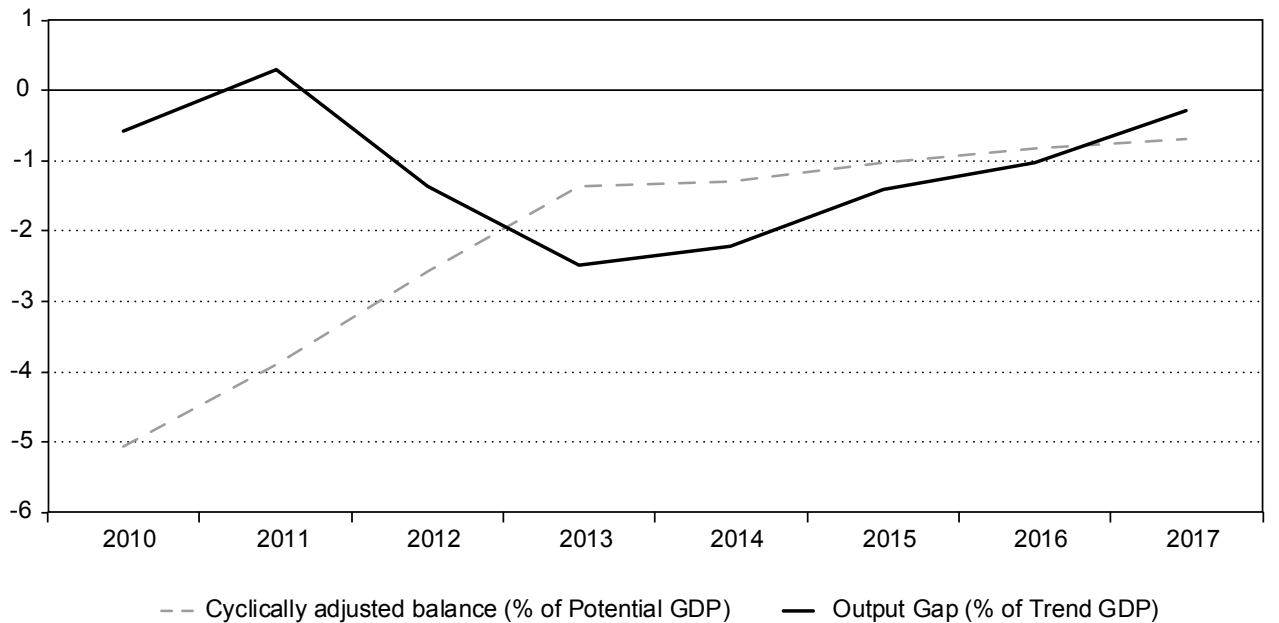

NOTA: Déficit ajustado por el ciclo estructural y Output Gap en la eurozona. FUENTE: AMECO, FMI y Funcas.

Durante los años más duros (2011-2013), prácticamente todos los países adoptaron medidas de austeridad. De ahí que el déficit estructural se redujera en dos puntos porcentuales - cuando lo que se espera es un apoyo de la política fiscal en momentos de debilitamiento coyuntural- . Sin duda, el corte restrictivo de la política fiscal agudizó la recesión, sobre todo en los países más afectados por la rarefacción del crédito, como España 4 .

Por otra parte, al revés de lo que sería deseable, la recuperación se caracteriza por una relajación de los esfuerzos de consolidación. En una mayoría de países miembros, el déficit estructural se ha mantenido apenas sin cambios, o incluso se ha acrecentado, como en España. Esto se explica en parte por la necesidad de aprovechar la expansión para revertir algunas de

\footnotetext{
4 Para un análisis reciente por parte del BCE de las interacciones entre política fiscal y financiación de la economía, ver Marek JAROCIŃSKI y Bartosz MAĆKOWIAK (2017).
}

las restricciones aplicadas durante el período de recesión-compresión de gastos o (con menor frecuencia) elevación de impuestos.

Es significativo que el déficit acusó una fuerte contracción durante el período 2010-2012 en todos los países de la eurozona (en el caso de España, la reducción se dilató algo más). Durante ese período, el PIB de la eurozona paso de crecer un 2,1 por 100 en 2010, a caer un 0,9 por 100 en 2012 (una desaceleración de tres puntos porcentuales). Sin embargo, el proceso de consolidación fiscal se ha ralentizado durante la expansión, que ha ganado fuerza desde 2015.

Mientras tanto, los principales países europeos que no participan en la moneda única aumentaron su déficit (Dinamarca y Suecia) o lo mantuvieron (Reino Unido) durante el período 2010-2012. Por el contrario, la orientación de la política fiscal es generalmente restrictiva en esos países desde el inicio de la recuperación. Ahora el presupuesto arroja un superávit en Dinamarca y Suecia, y un déficit reducido en Reino Unido. 
El segundo problema radica en la asimetría en el modus operandi del Semestre Europeo con respecto a la política fiscal (Bofinger, 2018). Las instituciones europeas son relativamente exigentes frente a los países que requieren un ajuste fiscal. Sin embargo, se muestran más benévolas ante situaciones de excedente.

Por ello, ningún país quiere figurar en el pelotón de cola del ajuste fiscal para así evitar exponerse a procedimientos por déficits excesivos. En realidad, los países compiten por tener un déficit reducido. La consecuencia es que la política fiscal tiende a ser globalmente contractiva, especialmente en fases de recesión.

Ese sesgo deflacionista, que se deriva de las medidas europeas de seguimiento adoptadas durante la crisis de la deuda soberana, explica el incremento acusado del superávit de balanza corriente de la eurozona (Cuadro 1). En 2017, el superávit alcanzó cerca de 400.000 millones de euros, máximo de la serie histórica. Es decir, la eurozona se caracteriza por una insuficiencia de la inversión, con respecto al ahorro disponible. El desequilibrio es el más elevado de las principales economías del mundo, y constituye un foco de preocupación sobre la sostenibilidad del crecimiento —además de alimentar el discurso proteccionista del Gobierno americano-.

El contenido de recomendaciones del Semestre Europeo tiende también a ser asimétrico, por ser más contundentes por el lado de la moderación del gasto público que del incremento de los impuestos. El corte prescriptivo y pormenorizado de las observaciones del Semestre Europeo es llamativo y contrasta con la relativa ambigüedad de las recomendaciones en materia impositiva como instrumento de corrección de los desequilibrios. Esto es importante porque, en período de recesión, los ajustes mediante recortes de gasto tienden a incidir más en la economía que un aumento de impuestos, sobre todo sobre las rentas elevadas con menor propensión a consumir (Berger et al., 2018).

La experiencia relativamente exitosa de países como Portugal o (fuera del euro) Suecia, que han actuado mediante una mezcla de medidas de contención de

\begin{tabular}{|c|c|c|c|}
\hline \multicolumn{4}{|c|}{$\begin{array}{l}\text { BALANZA POR CUENTA CORRIENTE } \\
\text { (En miles de millones de dólares) }\end{array}$} \\
\hline & 2010 & 2017 & Diferencia \\
\hline 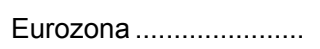 & $-7,7$ & $+442,4$ & $+450,0$ \\
\hline China & $+237,8$ & $+164,9$ & $-72,9$ \\
\hline Estados Unidos .............. & $-430,7$ & $-466,3$ & $-35,5$ \\
\hline Reino Unido...................... & $-92,3$ & $-106,7$ & $-14,4$ \\
\hline $\begin{array}{l}\text { Resto del mundo ........... } \\
\text { FUENTE: FMI y Funcas. }\end{array}$ & $+292,8$ & $-34,3$ & $-327,2$ \\
\hline
\end{tabular}

gasto y aumento de ingresos para contener el déficit estructural, muestra que no hay un solo camino para cumplir con los objetivos fiscales.

Consciente del riesgo que conllevan estas asimetrías, el Semestre Europeo se orienta hacia una nueva metodología. Ahora se enfatiza la evaluación como instrumento para mejorar la eficiencia del gasto, $y$ no necesariamente para aplicar nuevos recortes. Y se anima a crear autoridades fiscales independientes el Airef en España- para evaluar las diferentes opciones de corrección de los desequilibrios, sin necesariamente optar por una vía concreta, que podría chocar con las preferencias de cada país.

En tercer lugar, el actual sistema ha tenido poca incidencia sobre la trayectoria de la deuda pública a largo plazo, y ha operado de facto como un mecanismo de seguimiento del déficit a corto plazo ${ }^{5}$. El entorno de crecimiento moderado y de inflación baja o incluso negativa no ha sido propicio para la reducción del endeudamiento, pese a los avances en materia de déficit y los bajos tipos de interés (Gráfico 3). Asimismo, los Estados han tenido que asumir pasivos del sector privado (en el caso de España, en relación con las pérdidas del sector financiero y de las autopistas).

\footnotetext{
${ }^{5}$ Este es claramente el caso de España (TORRES y FERNÁNDEZ, 2018).
} 
GRÁFICO 3

LA DEUDA HA CRECIDO MÁS EN LA EUROZONA QUE FUERA DE ELLA

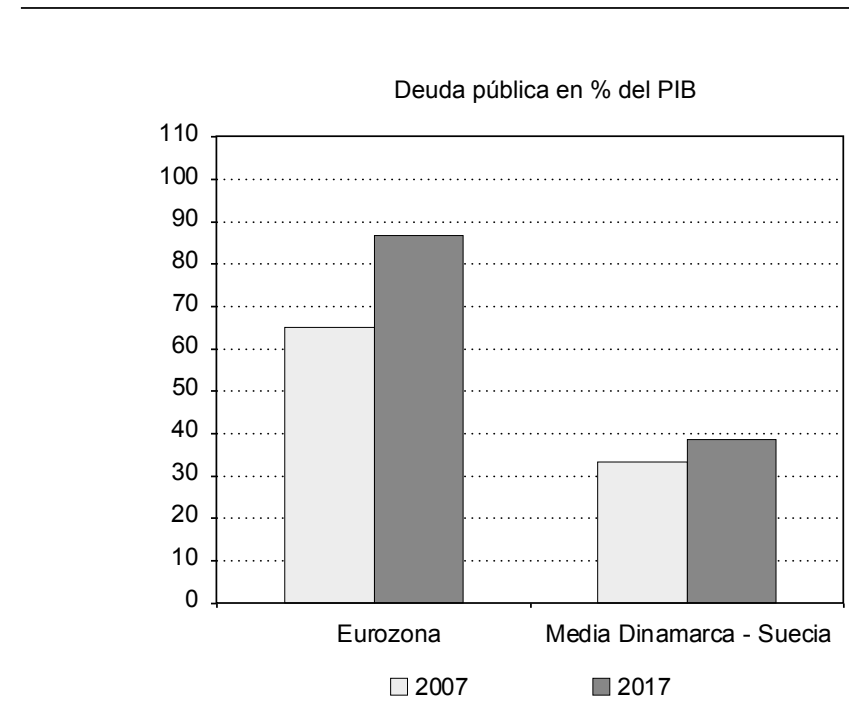

FUENTE: EUROSTAT, BEA, Haver Analytics y Funcas.

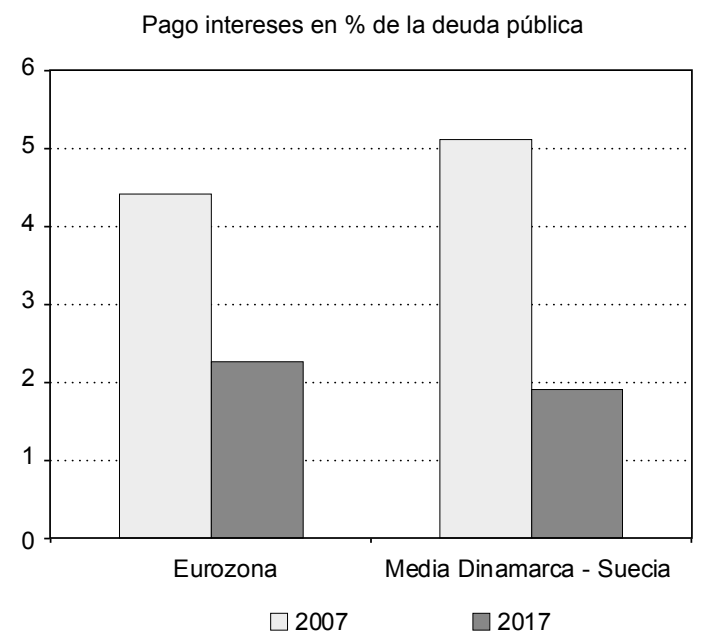

FUENTE: EUROSTAT, AMECO, Haver Analytics y Funcas.
Por otra parte, los activos de las Administraciones Públicas no se tienen en cuenta. Un endeudamiento que sirve para potenciar el crecimiento potencial del país, por ejemplo aumentando el capital tecnológico o mejorando las infraestructuras que dan sustento al crecimiento, es asumible por la economía. Sin embargo, en una mayoría de países, la acumulación de pasivos se ha producido a la vez que disminuía el esfuerzo inversor.

En un contexto de baja inversión pública y débil crecimiento potencial, las cargas financieras que se derivan de la deuda serán difíciles de asumir, especialmente cuando repunten los tipos de interés.

\section{Potenciación de una política fiscal europea}

Además de un mecanismo de coordinación de políticas fiscales nacionales, Europa dispone también de instrumentos presupuestarios supranacionales. Los fondos estructurales, el plan de inversiones (Plan Juncker) y, sobre todo, la acción del BCE contienen elementos estabilizadores. Sin embargo, los actuales instrumentos parecen insuficientes.

En primer lugar, porque no están a la altura de eventuales choques asimétricos. La reunificación de Alemania o el estallido de la burbuja inmobiliaria en España son riesgos específicos que requieren una respuesta macroeconómica contundente y adaptada a cada situación. Ante este tipo de contingencias, la historia económica apunta a alteraciones en el tipo de cambio como mecanismo de ajuste —algo por definición imposible en la eurozona (a menos de tomar la arriesgada decisión de reintroducir una moneda nacional)—.

La puesta en marcha de medidas monetarias y fiscales es otra opción, complementaria a los ajustes cambiarios. Al haber abandonado el arma monetaria en manos del BCE, que por su naturaleza no puede discriminar entre países miembros, el único margen de maniobra reside en la política fiscal. Sin embargo, la política fiscal está condicionada por los criterios establecidos en el Pacto de Estabilidad y Crecimiento, 


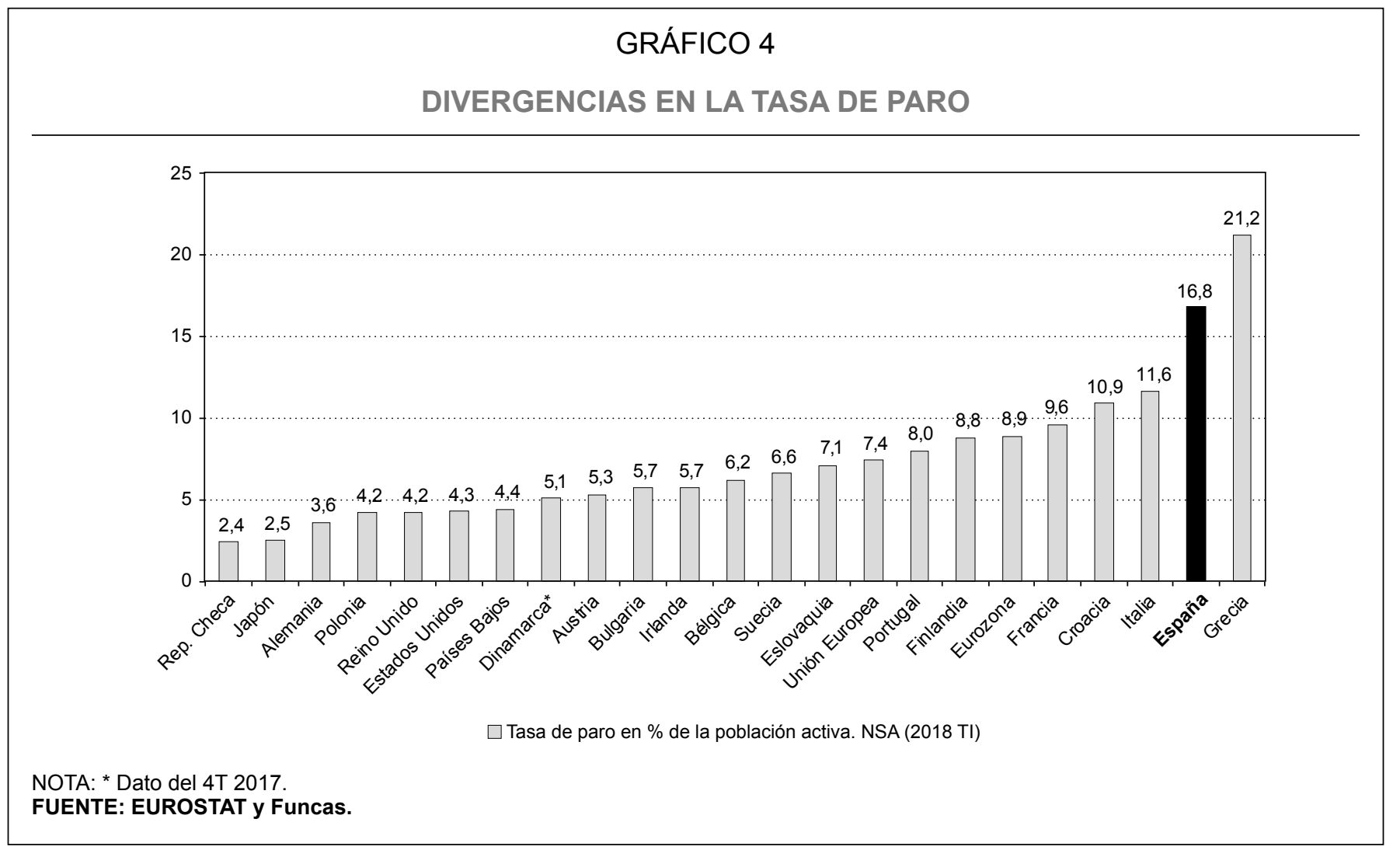

que en la práctica limitan su capacidad de respuesta a choques asimétricos.

Tampoco la coordinación de las políticas fiscales nacionales, aún con las mejoras antes mencionadas, es suficiente para hacer frente a choques asimétricos.

En segundo lugar, los instrumentos europeos no han conseguido detener la divergencia en el seno de la eurozona. Es un hecho que la convergencia se ha detenido estos últimos años, como lo muestra el diferencial de tasas de paro (Gráfico 4). Algunas de las economías más avanzadas, como Alemania y Países Bajos, experimentan tasas de crecimiento superiores a la media. Otras, que ya partían con desventaja, se han estancado. La situación es especialmente preocupante para Grecia e Italia. Pero la economía francesa también parece haberse desligado de la vecina Alemania.

Una comparación en términos de productividad y de inversión conduce a conclusiones similares. El retraso de algunos países es especialmente acusado, cuestionando la capacidad para aprovechar el tirón de las nuevas tecnologías y de la revolución de la inteligencia artificial. Los indicadores de $\mathrm{I}+\mathrm{D}$, patentes y utilización de los robots también dibujan un panorama de divergencias que plantea un reto para la estabilidad de la eurozona.

Finalmente, desde la construcción de la eurozona, los Estados carecen del apoyo monetario para resolver crisis de liquidez —el llamado "pecado original» del euro (Bofinger, 2018). En los países que no forman parte de la eurozona y en EE UU el Tesoro público tiene el respaldo del banco central para hacer frente a eventuales crisis de liquidez. Estas se producen, por ejemplo, cuando los flujos de capital se detienen (sudden stops) o por otras razones que no tienen que ver con la sostenibilidad de las finanzas públicas.

Esta circunstancia se produjo en el momento de la crisis de deuda soberana de 2010 , y provocó la recaída 


\section{GRÁFICO 5}

\section{DEUDA DE LAS ADMINISTRACIONES PÚBLICAS ESPAÑOLAS COLOCADA EN EL} EUROSISTEMA (BCE) Y EN EL RESTO DE SECTORES

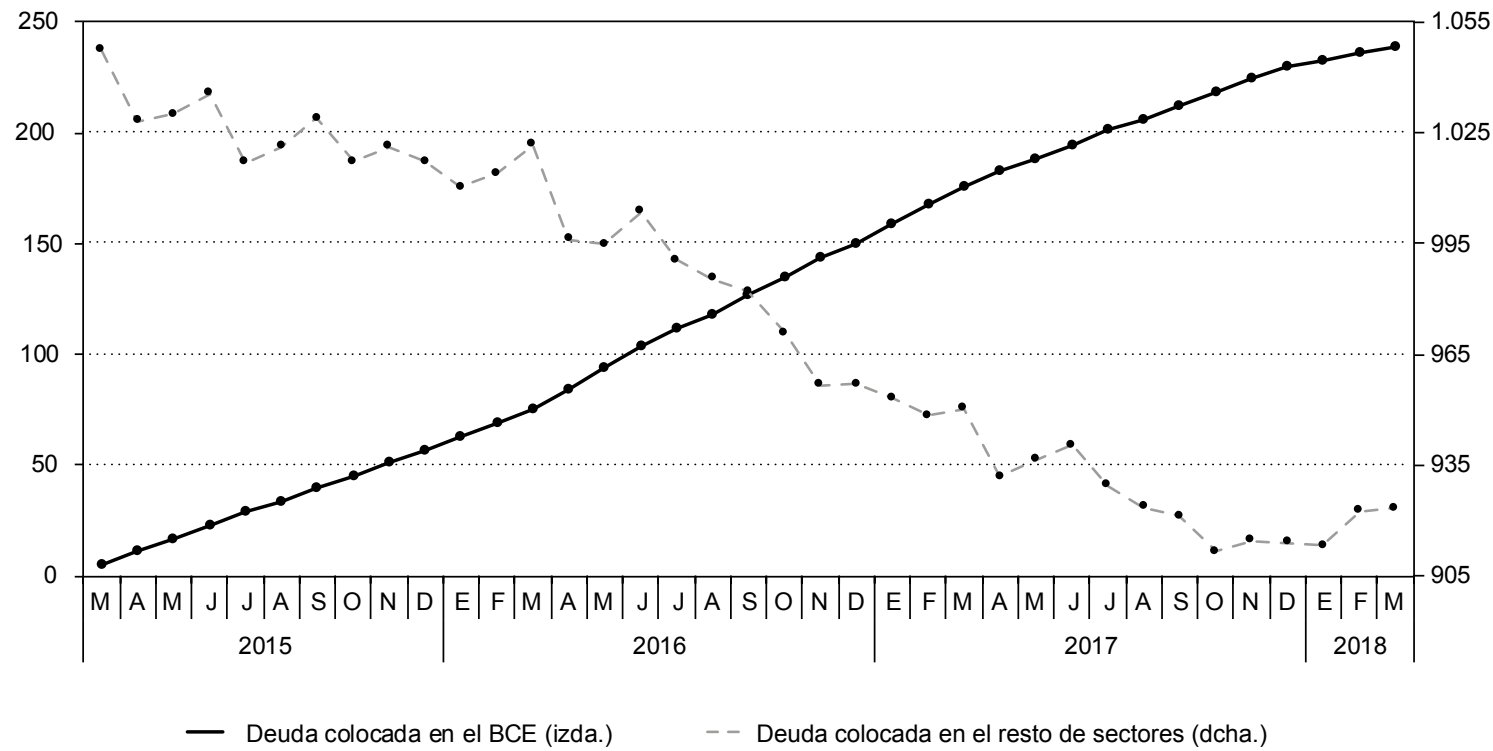

FUENTE: BCE, BDE y Funcas.

en la crisis de la economía española. Gracias a la acción del BCE, y en especial el programa de compra de títulos de deuda pública, el riesgo de crisis de liquidez se ha atenuado, sin desaparecer. Así pues, la normalización de la política monetaria del BCE plantea un serio desafío, sobre todo para países que soportan una deuda pública elevada como España, y teniendo en cuenta que cerca del 20 por 100 de esa deuda está colocada en el Eurosistema (Gráfico 5).

Para evitar nuevas crisis de liquidez, es crucial completar la unión financiera, y así reducir la exposición de los balances bancarios a la deuda pública nacional.

Pero también se necesita un mecanismo ordenado para hacer frente a crisis de solvencia, que se producen cuando un Estado no puede asumir la carga de su deuda. Es importante que tales crisis se resuelvan con celeridad, para evitar un rápido deterioro de las expectativas de los mercados, que acaben contagiando al resto de países. Para ello se ha creado el Mecanismo Europeo de Estabilidad. Sin embargo, la regla de unanimidad y de consulta de todos los Parlamentos nacionales representa una importante fuente de inercia, que dificulta su tarea. Por ello, algunos analistas abogan por transformar el MEDE en fondo monetario europeo, con capacidad de decisión, que dependa de una mayoría cualificada.

\section{Opciones de reforma}

\section{Mejora de los instrumentos de coordinación de políticas fiscales nacionales}

La Unión Europea, consciente del riesgo de un estancamiento de la unión económica y monetaria, ha empezado a corregir el sistema (Buti et al., 2018). Pero los esfuerzos ya realizados deben ser completados por reformas de más calado. 
En primer lugar, se necesita una estrategia creíble de reducción de la deuda a medio plazo. Al concentrar los esfuerzos en el déficit, no se tiene en cuenta el contenido de las medidas y su impacto sobre el crecimiento, la base fiscal y los déficits futuros.

Otra manera de redefinir el objetivo consistiría en la inclusión de los activos generados por las Administraciones Públicas. Las inversiones en nuevas tecnologías y otros activos intangibles, aunque provoquen un déficit a corto plazo, promueven el potencial productivo y la capacidad para aumentar la recaudación en el futuro. Lo mismo ocurre con las inversiones públicas en infraestructuras, en la medida en que estas responden a criterios racionales. En algunos países como Italia, la contención del déficit se ha conseguido en detrimento del equipamiento del país, del sistema educativo y del capital científico. Una formulación amplia de los objetivos fiscales obligaría a plantear otras decisiones en materia presupuestaria.

En segundo lugar, la política fiscal debe tener en cuenta el ciclo económico y en especial evitar recortes de gasto o aumentos de impuestos en períodos recesivos. Una senda procíclica de reducción de los desequilibrios es contraproducente desde el punto de vista coyuntural, además de provocar daños innecesarios en la creación de empleo y debilitar el potencial de crecimiento. $Y$ por otra parte retrasa el cumplimiento de los objetivos de deuda, tal y como definimos más arriba.

Una lógica contracíclica también implica que los criterios deberían respetarse con más determinación durante las épocas de expansión.

En tercer lugar, conviene priorizar el desarrollo institucional, en sustitución de un seguimiento europeo pormenorizado, y percibido como demasiado invasivo, de la política fiscal de cada país. Es importante que los procesos comunitarios se articulen con las instituciones democráticas, que son las que tienen que tomar decisiones en función del contexto propio a cada país.

Para ello sería de gran utilidad la creación de organismos independientes de seguimiento y de evaluación a nivel nacional, o su potenciación donde ya existen.
Estos organismos podrían convertirse en un importante eslabón en el sistema europeo de coordinación. La evaluación de las políticas impositivas y de gasto son actividades notoriamente insuficientes en la mayoría de países europeos. En el caso de España, el Airef es un primer paso en la buena dirección, que debería inspirar nuevas iniciativas (hoy por hoy huérfanas, tras la desaparición de la AEVAL). No se trata solo de asegurar un control administrativo de las políticas, algo que ya existe, sino de realizar una evaluación funcional de la ejecución de los programas con respecto a los objetivos perseguidos.

Finalmente, es importante orientarse hacia una mayor simetría entre países excedentarios y deficitarios, para contener el sesgo deflacionista y el carácter procíclico de la política fiscal. A falta de un presupuesto europeo anticíclico, la responsabilidad de incentivar la economía en fases recesivas recae en los Gobiernos, lo que en la práctica significa en aquellos países que disponen de más margen presupuestario. Este tipo de ajustes asimétricos son compatibles con los criterios de deuda, en la medida que los países con superávit apoyan la inversión y el capital productivo.

\section{Creación de un instrumento europeo de política fiscal que responda a los desafíos}

La creación de un instrumento de gestión anticíclica a nivel europeo resolvería buena parte de los defectos del actual sistema. Por una parte, permitiría hacer frente directamente a choques asimétricos, y así complementar las políticas fiscales nacionales. Para ser eficaz, este instrumento debería activarse con celeridad ante estos choques y a la vez ser dotado de recursos financieros suficientes (Claeys et al., 2016). En EE UU, el American Investment Act se puso en marcha desde el inicio de la crisis, y jugó un papel decisivo en la recuperación. Sus recursos alcanzaron en torno al 5 por 100 del PIB, repartidos sobre un período de tres años.

Existen varias opciones de reforma, pero todas ellas requieren de una capacidad fiscal común a nivel 
comunitario, a lo que habría que añadir la aplicación de condicionalidades para reducir el riesgo moral ${ }^{6}$.

La primera de esas opciones consiste en crear un fondo de inversión, similar al instrumento americano. Existe un precedente, el Plan Juncker. Sin embargo, este instrumento se limita a coordinar inversiones nacionales, complementadas por una modesta contribución europea mediante el Banco Europeo de Inversiones. También, en principio, el Plan Juncker se distribuye proporcionalmente al peso de cada país. El nivel de desempleo o el contexto coyuntural de los diferentes territorios influyen poco en la distribución geográfica del Plan, lo cual es lógico, por no tratarse de una política supranacional.

Por el contrario, la creación de un mecanismo a nivel comunitario respondería directamente a la situación específica de cada país. Para hacerlo aceptable por todos los socios, se requiere de criterios que: i) reconozcan explícitamente que el fondo no se dedica a ningún país en concreto, sino a cualquier economía que experimente dificultades coyunturales; ii) impongan condicionalidades (reformas que mejoren el funcionamiento de los mercados, política industrial de regeneración del tejido productivo, etc.); y iii) prevengan la relajación del esfuerzo propio de inversión (y así evitar el efecto sustitución de una política nacional por un instrumento europeo, que se interpretaría como un subsidio).

Una segunda opción consiste en un fondo de complemento europeo al sistema nacional de desempleo, que se añadiría al seguro de paro ya existente en cada país. El sistema se activaría automáticamente cada vez que la tasa de paro rebase cierto umbral. Por ejemplo, tres puntos más que la tasa media de paro observada en un ciclo completo. La principal ventaja de este método es su rápida reacción al ciclo —sobre todo en comparación con el fondo de inversión, que necesariamente requiere de un período relativamente largo para ser operacional.

\footnotetext{
6 Para una discusión exhaustiva de las diferentes opciones, así como la interacción con los incentivos del sistema, ver BÉNASSY-QUÉRÉ et al. (2018), así como la crítica de BINI SMAGHI (2018).
}

Por otra parte, el fondo de complemento europeo de desempleo contiene elementos disuasivos, que limitan reacciones oportunistas de países que recortarían sus prestaciones para beneficiarse de la ayuda comunitaria (riesgo moral). Por sí sola, la agravación del desempleo conlleva un deterioro de la imagen de los Gobiernos ante su opinión pública. $Y$ un recorte de las prestaciones sin duda perjudicaría aún más esa imagen.

Otra manera de reducir el riesgo moral es imponiendo condicionalidades. Por ejemplo, los países que recurren al fondo deberían reformar el mercado laboral para atajar la temporalidad en el empleo, un importante factor de precariedad y de paro de larga duración, o mejorar la efectividad de las políticas activas de empleo. Incluso se podría dedicar una parte de la ayuda a la inversión en aquellos programas de activación que a priori parecen ser más eficientes (fortalecimiento de los servicios públicos de empleo, adopción de dispositivos bien diseñados y evaluados de formación para parados, seguimiento de los resultados de las políticas de colocación de parados y de los planes de acción individuales).

El principal inconveniente de un fondo de estas características es la previsible reticencia de los países que se han acercado al pleno empleo. El fondo se podría diseñar de manera que, en algún momento de la historia reciente, todos los países se hubieran beneficiado de él. El umbral del 3 por 100 aquí propuesto cumple ese criterio. Para contener esas reticencias y apelar a la solidaridad, se podría dar un primer paso reservando el fondo a los jóvenes. Esta sería también una manera de avanzar con un compromiso ampliamente compartido, y en coherencia con la Garantía Joven —un programa que ya contaba con financiación europea-.

Finalmente, algunos expertos abogan por un fondo sin criterios específicos de gasto. Se podría utilizar tanto para fomentar la inversión como para complementar ingresos, incentivar la creación de empresas o evitar su quiebra. Este sistema ofrece más flexibilidad, pudiéndose adaptar a las prioridades de cada país. Por ejemplo, a principios de los años 2000 la economía finlandesa registró un duro golpe como consecuencia 
de la recesión en la vecina Rusia. Sin embargo, el debilitamiento se concentró en un sector, lo que exigió un tratamiento específico, con una mezcla de medidas de reconversión, formación y reciclaje, y subsidios temporales a actividades por lo demás rentables.

La financiación es una cuestión crucial que condiciona la capacidad de respuesta europea. La emisión de títulos de deuda europeos resulta especialmente atractiva, tanto por su flexibilidad como por el excelente rating de las instituciones comunitarias. Esta solución tiene la ventaja adicional de no detraer recursos de los países que más los necesitan.

Los eurobonos, garantizados conjuntamente por los Tesoros europeos, servirían para financiar el fondo europeo de gestión macroeconómica. Algunos países se muestran reticentes a su lanzamiento, por el peligro que supone abrir una nueva fuente de deuda. Incluso consideran que su actual calificación crediticia refleja una gestión rigurosa de la hacienda pública. Al crear eurobonos, los mercados reconsiderarían su posición con respecto a los países que, por sí solos, consiguen equilibrar el presupuesto sin ayuda europea. Por el contrario, las maltrechas haciendas de otros países se beneficiarían del respaldo europeo, especialmente en fases recesivas. Una forma de contener ese riesgo es obligar a que los eurobonos que se emiten en fases de recesión se devuelvan en momentos favorables de coyuntura por los países que se beneficiaron de ellos.

$\mathrm{Si}$ el fondo europeo anticrisis consiste en un sistema complementario de desempleo, la financiación podría provenir de la puesta en común de una fracción de las cotizaciones sociales. Al no necesitar la emisión de eurobonos, el sistema no perjudicaría el ranking de los países virtuosos. Por otra parte, cada país tendría que realizar un esfuerzo adicional en momento de expansión para así ganar el derecho a movilizar recursos del sistema europeo en momentos de fuerte aumento del desempleo.

Finalmente, una participación de fuentes privadas de financiación es posible y deseable si Europa finalmente se decanta por un fondo europeo de inversión. Esta, que es la principal característica del Plan Juncker, se puede extender tanto en volumen como en la composición nacional de la financiación. En la actualidad, solo en una pequeña proporción los recursos son comunitarios. Esta proporción podría variar según la posición coyuntural de cada país. De esta forma se evitaría la actual situación de abundancia relativa del Plan Juncker en países que conocen un crecimiento elevado, coexistiendo con escasez de fondos en territorios que más los necesitan y que presentan oportunidades de inversión.

La convergencia requiere de cierta armonización cuando diferencias de legislación nacional pueden provocar una competencia desleal. Este es especialmente el caso en cuanto a la fiscalidad de las empresas. Las diferencias de base fiscal, el tratamiento de los royalties y el complejo entramado de deducciones fiscales dan lugar a transferencias de ingresos en el seno de los grupos empresariales, que acaban por erosionar la base impositiva. Esta situación es perjudicial para las arcas públicas de todos los Estados, en especial aquellos que se muestran más estrictos en los criterios de equidad entre personas físicas y morales, y que luchan con más ahínco contra las prácticas de evasión.

Por otra parte, la política comunitaria podría facilitar la convergencia real de las economías. Algunos analistas abogan por la introducción de incentivos a la reforma o de apoyo técnico por parte de la Comisión. La cuestión es compleja, por interferir con las preferencias de cada país. Por ejemplo, no existe un único modelo exitoso de reforma del mercado laboral. Se puede facilitar la contratación y a la vez proporcionar protección social (modelo holandés), o se puede priorizar la flexibilidad (modelo anglosajón). Los efectos sobre el reparto de la renta son distintos, como también lo son las implicaciones en términos de gasto público. Pero la evidencia empírica muestra que las tasas de desempleo son similares en ambos sistemas. El papel de Europa es ilustrar las tensiones entre distintos objetivos y evaluar las consecuencias sobre el empleo y la convergencia - pero no parece lógico que se sustituya a los países en la elección de su modelo- . 
En suma, la reforma de la política fiscal europea, tal y como se presenta en este y otros análisis, necesariamente implica un abandono de parte de la soberanía nacional, lo que plantea la cuestión del control democrático. Por ello, varios expertos proponen el nombramiento de un Ministro de Hacienda europeo, responsable ante el Europarlamento. Además, conviene delimitar las competencias europeas -fundamentalmente con respecto a la gestión del instrumento fiscal supranacional y la vigilancia de los equilibrios macroeconómicos nacionales-. Las instituciones de gobernanza de cada país seguirían siendo responsables de sus presupuestos.

En definitiva, existen opciones de reforma y de potenciación de una política fiscal europea. Todas ellas suponen un abandono de soberanía nacional, a cambio de una mayor eficacia y más solidaridad. Ese es el dilema.

\section{Referencias bibliográficas}

[1] BEGG, I. (2018). Reform of the EU Fiscal Framework. Funcas Europe (Forthcoming).

[2] BÉNASSY-QUÉRÉ, A; BRUNNERMEIER, K.; ENDERLEIN, M.H.; FARHI, E.; FRATZSCHER, M.; FUEST, P-O; GOURINCHAS, C.; MARTIN, C.; PISANI-FERRY, J.; REY, H.; SCHNABEL, I; VÉRON, N.; WEDER Di MAURO, B. y ZETTELMEYER, J. (2018). «Reconciling Risk Sharing with Market Discipline: A Constructive Approach to Euro Area Reform». CEPR Policy Insight, $\mathrm{n}^{\circ} 91$.
[3] BERGER, H.; DELL'ARICCIA, G. y OBSTFELD, M. (2018). Revisiting the Economic Case for Fiscal Union in the Euro Area, IMF Research Department.

[4] BINI SMAGHI, L. (2018). A Stronger Euro Area through Stronger Institutions, https://voxeu.org/article/stronger-euroarea-through-stronger-institutions

[5] BOFINGER, P. (2018). Euro Area Reform: No Deal is Better than a Bad Deal. Vox EU. En https://voxeu.org/article/ cepr-policy-insight-91-no-deal-better-bad-dealChtps://voxeu. org/article/cepr-policy-insight-91-no-deal-better-bad-deaRal

[6] BUTI, M.; GIUDICE, G. y LEANDRO, J. (2018). Deepening EMU Requires a Coherent and Well-sequenced Package. Vox EU, https://voxeu.org/article/deepening-emu-requires-coherent-and-well-sequenced-package

[7] CLAEYS, G.; DARVAS, Z.M. y LEANDRO, A. (2016). A Proposal to Revive the European Fiscal Framework. Bruegel Policy Contribution.

[8] EUROPEAN FISCAL BOARD (2017). Assessment of the Prospective Fiscal Stance Appropriate for the Euro Area, June.

[9] FATÁS, A. y SUMMERS, L.H. (2017). «The Permanent Effects of Fiscal Consolidations». NBER Working Paper Series $\mathrm{n}^{\circ} 22374$. En http://www.nber.org/papers/w22374

[10] FONDO MONETARIO INTERNACIONAL (2009). G20 London Summit - Leaders' Statement, 2 April 2009, www.imf.org/external/np/sec/pr/2009/pdf/g20_040209.pdf

[11] JAROCIŃSKI, M. y MAĆKOWIAK, B. (2017). «Monetary-fiscal Interactions and the Euro Area's Vulnerability», ECB Research Bulletin, $\mathrm{n}^{\circ}$ 36. https://www.ecb.europa.eu/pub/economic-research/resbull/2017/html/ecb.rb170629.en.html

[12] TORRES, R. y FERNÁNDEZ, M.J. (2018). «The Spanish Economy: Scenarios for 2018-2020», Funcas, Spanish Economic and Financial Outlook, vol. 7, $\mathrm{n}^{\circ}$ 2, marzo.

[13] WIESER, T. (2018). Fiscal Rules and the Role of the Commission, Bruegel. 